\title{
Analysis of Rumor Spreading with 2-pull or 3-pull Operations
}

\author{
Yves Mocquard, Bruno Sericola \\ Inria, Univ. Rennes, CNRS, IRISA \\ Inria, Campus de Beaulieu, France \\ firstname.lastname@inria.fr
}

\author{
Emmanuelle Anceaume \\ CNRS, Univ. Rennes, Inria, IRISA \\ IRISA, Campus de Beaulieu, France \\ emmanuelle.anceaume@irisa.fr
}

\begin{abstract}
In this paper, we analyze a new asynchronous rumor spreading protocol to deliver a rumor to all the nodes of a largescale distributed network. This protocol relies on successive pull operations involving $k$ different nodes, with $k=2$ or $k=3$, and called $k$-pull operations. Specifically during a $k$-pull operation, an uninformed node $a$ contacts $k-1$ other nodes at random in the network, and if at least one of them knows the rumor, then node $a$ learns it. We perform a detailed study in continuous-time of $\Theta_{k, n}$, the total time needed for all the $n$ nodes to learn the rumor.

We obtain, for $k \in\{2,3\}$, the mean value, the variance and the distribution of $\Theta_{k, n}$ together with their asymptotic behavior when the number of nodes $n$ tends to infinity.
\end{abstract}

Keywords : Rumor spreading time; $k$-pull protocol; Poisson Process; Markov chain; Asymptotic analysis

\section{INTRODUCTION}

Randomized rumor spreading or gossiping is an important communication schema that allows the dissemination of information in large-scale and open networks. A large-scale and open network comprises a collection of sequential computing entities (i.e., processes, processors, nodes, agents, peers) that join and leave the system at any time, and communicate with one another by exchanging messages. Randomized rumor spreading was initially proposed by Deemers et al. [8] for the update of a database replicated at different sites, and has then been adopted in many applications due to its robustness and simplicity. In contrast to reliable communication broadcast schemas that must provide agreement on the broadcast value with possibly additional ordering guarantees on the delivery of updates from different sources, a randomized rumor spreading schema guarantees that all recipients of the broadcast value receive it only with some probability. A randomized spreading rumor protocol describes the rules required for one or more pieces of information known to an arbitrary node in the network to be spread to all the nodes of the network. The push and pull protocols are the basic operations nodes use to propagate an information over the entire network [8], [11]. With the push operation, an informed node contacts some randomly chosen node and sends it the rumor, while with the pull operation, an uninformed node contacts some random node and asks for the rumor. The same node can perform both operations according to whether it knows or not the rumor, which corresponds to the push-pull protocol, or performs only

978-1-7281-8326-8/20/\$31.00 @2020 IEEE one, either a pull or push operation, which corresponds to the pull or push protocols respectively. One of the important questions raised by these protocols is the spreading time, that is the time it needs for the rumor to be known by all the nodes of the network.

Several models have been considered to answer this question. The most studied one is the synchronous model. This model assumes that all the nodes of the network act in synchrony, which allows the algorithms designed in this model to divide time in synchronized rounds. During each synchronized round, each node $a$ of the network selects at random one of its neighbor $b$ and either sends to $b$ the rumor if $a$ knows it (push operation) or tries to get the rumor from $b$ if $b$ knows the rumor (pull operation). In the synchronous model, the spreading time of a rumor is defined as the number of synchronous rounds necessary for all the nodes to know the rumor. When the underlying graph is complete, it has been shown by Frieze [15] that the ratio of the number of rounds over $\log _{2}(n)$ converges in probability to $1+\ln (2)$ when the number $n$ of nodes in the graph tends to infinity. Further results have been established (see for example [20], [26] and the references therein), the most recent ones resulting from the observation that the rumor spreading time is closely related to the conductance of the graph of the network, see [16]. Investigations have also been done in different topologies of the network as in [3], [7], [14], [24], in the presence of link or nodes failures as in [13], in dynamic graphs as in [4] and spreading with node expansion as in [17]. Another alternative consists for the nodes to make more than one call during the push or pull operations [25]. This alternative is of particular interest since it does not require any particular network structure. The synchronous case has been tackled in $[25]$ where the authors show that the pushpull protocol takes $O(\log n / \log \log n)$ rounds in expectation assuming that nodes can connect to a random number neighbor following a specific power law during each single round.

In large scale and open networks, assuming that all nodes act synchronously is a very strong assumption. Several authors, including [1], [10], [18], [22], [27]), suppose that nodes asynchronously trigger operations with randomly chosen nodes. In [27], the authors model a multiple call by tuning the clock rate of each node with a given probability distribution. Some authors have focused on the message complexity by optimizing the network structure [9], [11], [18], [23]. For 
instance, in [9], the authors show that the asynchronous pushpull protocol spreading time in a preferential attachment graph is in $O(\sqrt{\log n})$. Another way of limiting the number of interactions is by finely tuning the push and pull operations to take advantage of both of them as achieved for example in $[8],[12]$, or by relying on a central authority to coordinate the work (e.g, [5]).

The asynchronous gossip protocol is usually modeled by a time-continuous stochastic (Markovian) process [1], [10], [18], [22], [27]. This type of stochastic processes belongs to the death process category, which has many applications in demography, queuing theory, performance engineering, epidemiology, biology and many other distributed applications. For instance, in [6], an analysis of the SI (Susceptible-Infected) model -corresponding to an asynchronous push-pull modelallows us in some cases to explicit the state probabilities by using the Laplace transform on the Kolmogorov forward equation. However, these techniques prove ineffective when the transition rate is non-linear (Laplace transform inversion becomes a tricky exercise). Most of the results then focus on studying the asymptotic behavior by using martingale techniques to obtain a law of large numbers or a central limit theorem [2]. However, one may study transient behaviour using a coupling technique [21]. The principle is to look for simpler linear processes that serve as bounds to the initial processes.

The pull algorithm attracted very little attention because this operation was long considered inefficient to spread a rumor within a large scale network [29]. It is actually very useful in systems fighting against message saturation (see for instance [31]). The ineffectiveness of the pull protocol stems from the fact that it takes some time before the rumour reaches a phase of exponential growth. Conversely, the push protocol initiates the rumor very quickly but then struggles to reach the few uninformed nodes. In this paper, we seek to counterbalance the slow initiation of rumour spreading by increasing the chances of learning the rumour with each call. We will analyze $\Theta_{k, n}$, the total time needed for all the $n$ nodes of the system to learn the rumor initially known by a single random node in the system. Our analysis will explicitly show that calling the rumor from 2 nodes in parallel rather from a single one performs better.

The remainder of this paper is organized as follows. In Section III we present both the discrete-time and the continuoustime models of the $k$-pull asynchronous rumor spreading protocol. We evaluate in Section III the first two moments of the rumor spreading time. We show for instance that the asymptotic expected rumor spreading time when uniformed nodes call the rumor from 2 random nodes (i.e. when $k=3$ ), is equivalent to $3 \ln (n) /(2 \lambda)$, where $\lambda$ is the rate of the exponentially distributed local clock of each uniformed node, when $n$ tends to infinity. Note that when a uniformed node calls the rumor from a single node (i.e. when $k=2$ ), this asymptotic expectation is equivalent to $2 \ln (n) / \lambda$. The variance of $\Theta_{k, n}$ and its limiting value are analyzed in Section III-B This analysis shows that calling two random nodes instead of a single one at each operation provides a smaller standard deviation. We finally analyze in Section IV the probability distribution of the rumor spreading time and provide its exact limiting value when $n$ tends to infinity. Section $\mathrm{V}$ concludes.

\section{THE MODEL}

We recall in the next subsection the discrete-time model of the $k$-pull rumor spreading. This model which has been analyzed in [28] is important because the continuous-time model of the $k$-pull rumor spreading is based on the discretetime model, even if the analysis is, as we will see, much more complicated and needs more refined results in continuous-time.

\section{A. The discrete-time model}

A complete network populated by $n$ nodes is considered. Nodes are anonymous (i.e. they do not carry any identifier). Each node may be asked for a piece of information (rumor) initiated by any other node of the system. The algorithm starts with a single node informed of the rumor. At each discrete time $m \geq 1$, a single uninformed node $s$ contacts $k-1$ distinct nodes, chosen at random uniformly among the $n-1$ other nodes. If at least one of these $k-1$ contacted nodes knows the rumor then, node $s$ learns it. Otherwise nothing happens. This is a $k$-pull operation.

Analysis of this $k$-pull model is achieved by introducing the discrete-time stochastic process $Y=\left\{Y_{m}, m \geq 0\right\}$ where $Y_{m}$ represents the number of informed nodes at time $m$. Stochastic process $Y$ is a discrete-time homogeneous Markov chain with $n$ states where states $1, \ldots, n-1$ are transient and state $n$ is absorbing. From the description of the protocol, when the Markov chain $Y$ is in state $i$ at time $m$, then at time $m+1$, either it remains in state $i$ if none of the $k-1$ chosen nodes were informed of the rumor or it transits to state $i+1$ if at least one of the $k-1$ chosen nodes were informed of the rumor. Let $P$ denote the transition probability matrix of Markov chain $Y$. The non zero entries of matrix $P$ are thus $P_{i, i}$ and $P_{i, i+1}$, for any $i=1, \ldots, n-1$. Obviously, we get, for any $i=$ $1, \ldots, n-1, P_{i, i}=1-P_{i, i+1}$, which is given by

$$
P_{i, i}=\left\{\begin{array}{cl}
\frac{\left(\begin{array}{c}
n-1-i \\
k-1
\end{array}\right)}{\left(\begin{array}{l}
n-1 \\
k-1
\end{array}\right)} & \text { if } i \leq n-k \\
0 & \text { otherwise. }
\end{array}\right.
$$

Indeed, given that $Y_{t}=i$, i.e. when $i$ nodes are informed of the rumor at time $t$, we have $Y_{t+1}=i$ if and only if, at time $t+1$, the set of $k-1$ chosen nodes (i.e. $k-1$ among $n-1$ ) must be chosen among the $n-1-i$ non informed nodes.

Let $T_{k, n}$ be the random variable defined by

$$
T_{k, n}=\inf \left\{t \geq 0 \mid Y_{m}=n\right\},
$$

which represents the spreading time, that is the total number of $k$-pull operations needed for all the nodes in the network to know the rumor. 
The spreading time distribution can thus be expressed as a sum of independent random variables $S_{k, n}(i)$, where $S_{k, n}(i)$ is the sojourn time of Markov chain $Y$ in state $i$. For all $i=$ $1, \ldots, n-k, S_{k, n}(i)$ follows a geometric distribution with parameter $p_{k, n}(i)$, where

$$
p_{k, n}(i)=1-P_{i, i}=1-\prod_{h=1}^{k-1}\left(1-\frac{i}{n-h}\right)
$$

and $S_{k, n}(i)=1$, for $i=n-k+1, \ldots, n-1$. Observe that for $i=n-k+1, \ldots, n-1$, we have $p_{k, n}(i)=1$. Thus $T_{k, n}$ verifies

$$
T_{k, n}=\sum_{i=1}^{n-1} S_{k, n}(i)=k-1+\sum_{i=1}^{n-k} S_{k, n}(i) .
$$

\section{B. The continuous-time model}

Based on this discrete-time model, a local clock following an exponential distribution with rate $\lambda$ is associated with each uniformed node of the system. Each time the clock of an uniformed node $s$ rings, this node contacts $k-1$, with $k \geq 2$, distinct nodes, chosen at random uniformly among the $n-1$ other nodes. If at least one of these contacted nodes knows the rumor, node $s$ learns it and clears its clock (i.e., $s$ remains contactable but does not contact any other node). As in the discrete-time case, we suppose that the $k$-pull operation is instantaneous, that is, the time for a node to contact $k-1$ other nodes and to receive their response takes no time. Since the clock of an uninformed node rings after a time exponentially distributed with rate $\lambda$, we naturally introduce the continuoustime Markov chain $Z=\left\{Z_{t}, t \geq 0\right\}$, where $Z_{t}$ represents the number of informed nodes at continuous-time $t \geq 0$. Specifically the transitions of $Z$ occur at successive instants $\tau_{0}=0, \tau_{1}, \ldots$, where the $\tau_{i}-\tau_{i-1}, i \geq 1$, are independent and exponentially distributed with rate $(n-i) \lambda p_{k, n}(i)$, where the $p_{k, n}(i)$ are given by (1). Hence the global clock of the process rings according to an exponential distribution whose rate is proportional to the amount of uniformed nodes. Note that a jump of process $Z$ corresponds to a state change of process $Y$.

Observe also that the continuous-time model of the rumor spreading corresponds to a physical time, that is the total amount of time needed for all the $n$ nodes to learn the rumor, while the discrete-time model stands for the total number of $k$-pull operations needed for all the $n$ nodes to learn the rumor.

We denote by $\Theta_{k, n}$ the random variable defined by

$$
\Theta_{k, n}=\inf \left\{t \geq 0 \mid Z_{t}=n\right\},
$$

which represents the total amount of time needed for all the nodes to know the rumor. The spreading time $\Theta_{k, n}$ can thus be expressed as a sum of independent and exponentially distributed random variables. Specifically, introducing the notation $U_{k, n}(i)=\tau_{i}-\tau_{i-1}$, for $i \geq 1$ with $\tau_{0}=0$, we have

$$
\Theta_{k, n}=\sum_{i=1}^{n-1} U_{k, n}(i)
$$

where $U_{k, n}(1), \ldots, U_{k, n}(n-1)$ are independent and $U_{k, n}(i)$ is exponentially distributed with rate $(n-i) \lambda p_{k, n}(i)$.

The authors of [28] used two technical lemmas to analyze the moments and the distribution of the rumor spreading time in the discrete-time case. These lemmas allowed them to provide lower and upper bounds of the probabilities $p_{k, n}(i)$. These bounds are sufficiently precise to deal with the sum of geometric random variables with parameters $p_{k, n}(i)$ but they are too wide to deal with the sum of exponential random variables with rates $(n-i) p_{k, n}(i)$, in the continuous-time case. We thus consider here a different method to analyze the problem when $k \in\{2,3\}$.

\section{MOMENTS OF THE RUMOR SPREADING TIME}

In this section, we analyze the two first moments of the rumor spreading time $\Theta_{k, n}$, for $k \in\{2,3\}$, when $n$ goes to infinity.

\section{A. Expected rumor spreading time}

Observing that $p_{k, n}(i)=1$ for $i=n-k+1, \ldots, n-1$, the expected value of the spreading time $\Theta_{k, n}$ is given, using Relation (2), by

$$
\begin{aligned}
\mathbb{E}\left(\Theta_{k, n}\right) & =\frac{1}{\lambda} \sum_{i=1}^{n-1} \frac{1}{(n-i) p_{k, n}(i)} \\
& =\frac{1}{\lambda} \sum_{i=1}^{n-k} \frac{1}{(n-i) p_{k, n}(i)}+\frac{1}{\lambda} \sum_{i=1}^{k-1} \frac{1}{i} .
\end{aligned}
$$

For $k=2$, we have $p_{2, n}(i)=i /(n-1)$ and using (3) we obtain

$$
\mathbb{E}\left(\Theta_{2, n}\right)=\frac{n-1}{\lambda} \sum_{i=1}^{n-1} \frac{1}{i(n-i)}
$$

For $k=3$, we have

$p_{3, n}(i)=\frac{i}{n-1}+\frac{i}{n-2}-\frac{i^{2}}{(n-1)(n-2)}=\frac{i(2 n-3-i)}{(n-1)(n-2)}$,

which leads, using (3), to

$$
\mathbb{E}\left(\Theta_{3, n}\right)=\frac{(n-1)(n-2)}{\lambda} \sum_{i=1}^{n-1} \frac{1}{i(n-i)(2 n-3-i)} .
$$

The asymptotic expected rumor spreading time is obtained in the following theorem. We denote by $H_{n}$ the Harmonic series defined, for every $n \geq 1$, by $H_{n}=\sum_{i=1}^{n} 1 / i$ and we recall that the Euler-Mascheroni constant $\gamma$ is given by $\gamma=\lim _{n}\left(H_{n}-\ln (n)\right)$, which is approximately equal to 0.5772156649 .

Theorem 1. We have

$$
\begin{aligned}
\mathbb{E}\left(\Theta_{2, n}\right) & \underset{n \rightarrow \rightarrow \infty}{\sim} \frac{2 \ln (n)}{\lambda} \\
\text { with } & \lim _{n \rightarrow \infty}\left(\mathbb{E}\left(\Theta_{2, n}\right)-\frac{2 \ln (n)}{\lambda}\right)=\frac{2 \gamma}{\lambda}
\end{aligned}
$$


and

$$
\begin{aligned}
\mathbb{E}\left(\Theta_{3, n}\right) & \underset{n \rightarrow \infty}{\sim} \frac{3 \ln (n)}{2 \lambda} \\
\text { with } & \lim _{n \rightarrow \infty}\left(\mathbb{E}\left(\Theta_{3, n}\right)-\frac{3 \ln (n)}{2 \lambda}\right)=\frac{3 \gamma-\ln (2)}{2 \lambda} .
\end{aligned}
$$

Proof. For $k=2$, using a partial fraction expansion in Relation (4), we easily get

$$
\begin{aligned}
\mathbb{E}\left(\Theta_{2, n}\right) & =\frac{n-1}{\lambda n}\left(\sum_{i=1}^{n-1} \frac{1}{i}+\sum_{i=1}^{n-1} \frac{1}{n-i}\right) \\
& =\frac{2(n-1) H_{n-1}}{\lambda n} \underset{n \rightarrow \infty}{\sim} \frac{2 \ln (n)}{\lambda} .
\end{aligned}
$$

By definition of the Euler-Mascheroni constant $\gamma$ we easily get the second result.

For $k=3$, in the same way, using a partial fraction expansion in Relation (5), we obtain

$$
\frac{(n-1)(n-2)}{i(n-i)(2 n-3-i)}=\frac{A_{n}}{i}+\frac{B_{n}}{n-i}+\frac{C_{n}}{2 n-3-i},
$$

where

$$
\begin{aligned}
A_{n} & =\frac{(n-1)(n-2)}{n(2 n-3)}, \quad B_{n}=\frac{(n-1)(n-2)}{n(n-3)} \quad \text { and } \\
C_{n} & =\frac{-(n-1)(n-2)}{(n-3)(2 n-3)} .
\end{aligned}
$$

It follows that

$$
\begin{aligned}
\mathbb{E}\left(\Theta_{3, n}\right)=\frac{1}{\lambda}\left[A_{n} \sum_{i=1}^{n-1} \frac{1}{i}\right. & +B_{n} \sum_{i=1}^{n-1} \frac{1}{n-i} \\
& \left.+C_{n} \sum_{i=1}^{n-1} \frac{1}{2 n-3-i}\right] \\
=\frac{1}{\lambda}\left[A_{n} H_{n-1}\right. & \left.+B_{n} H_{n-1}+C_{n}\left(H_{2 n-4}-H_{n-3}\right)\right] .
\end{aligned}
$$

Since $\lim _{n \longrightarrow \infty} A_{n}=1 / 2, \lim _{n \longrightarrow \infty} B_{n}=1, \lim _{n \longrightarrow \infty} C_{n}=-1 / 2$ and $\lim _{n \longrightarrow \infty}\left(H_{2 n-4}-H_{n-3}\right) \stackrel{n}{=} \ln (2)$, we easily get

$$
\mathbb{E}\left(\Theta_{3, n}\right) \underset{n \rightarrow \infty}{\sim} \frac{3 \ln (n)}{2 \lambda} .
$$

Again, by definition of the Euler-Mascheroni constant $\gamma$, we get the last result.

Figure 1 illustrates Relations 4 and 5 . It shows the benefit of calling two random and anonymous nodes at each pull operation instead of a single one. For instance, when $n=100,000$ nodes, it takes in average 17.79 time units for all the nodes to know the rumor with the 3-pull protocol while it takes 24.18 time units with the 2-pull protocol. Moreover, from Theorem 1 . we deduce that the difference $\mathbb{E}\left(\Theta_{2, n}\right)-\mathbb{E}\left(\Theta_{3, n}\right)$ tends to infinity when $n$ tends to infinity. Indeed, it is easily checked that

and that

$$
\mathbb{E}\left(\Theta_{2, n}\right)-\mathbb{E}\left(\Theta_{3, n}\right) \underset{n \rightarrow \infty}{\sim} \frac{\ln (n)}{2 \lambda}
$$

$$
\lim _{n \longrightarrow \infty}\left(\mathbb{E}\left(\Theta_{2, n}\right)-\mathbb{E}\left(\Theta_{3, n}\right)-\frac{\ln (n)}{2 \lambda}\right)=\frac{\gamma+\ln (2)}{2 \lambda} .
$$

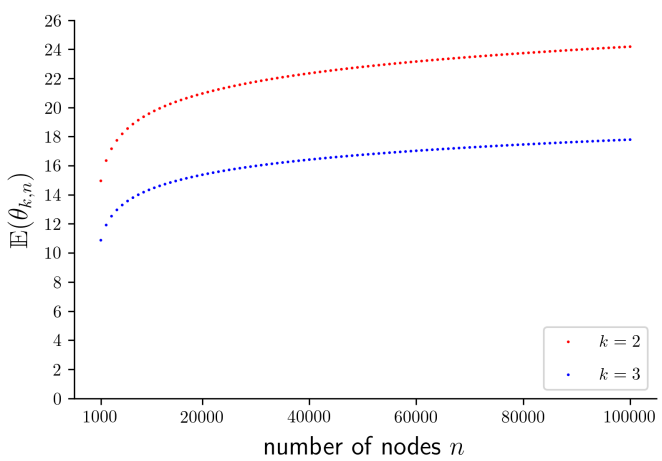

Fig. 1. Expected values $\mathbb{E}\left(\Theta_{2, n}\right)$ and $\mathbb{E}\left(\Theta_{3, n}\right)$ as a function of the number of nodes $n$.

\section{B. Variance of the rumor spreading time}

We consider now the variance of $\Theta_{k, n}$ and its limiting value when $n$ goes to infinity. Using Relation (2), the variance of $\Theta_{k, n}$ is given by

$$
\begin{aligned}
\operatorname{Var}\left(\Theta_{k, n}\right) & =\frac{1}{\lambda^{2}} \sum_{i=1}^{n-1} \frac{1}{(n-i)^{2}\left(p_{k, n}(i)\right)^{2}} \\
& =\frac{1}{\lambda^{2}}\left[\sum_{i=1}^{k-1} \frac{1}{i^{2}}+\sum_{i=1}^{n-k} \frac{1}{(n-i)^{2}\left(p_{k, n}(i)\right)^{2}}\right]
\end{aligned}
$$

For $k=2$, since $p_{2, n}(i)=i /(n-1)$, we obtain using (7)

$$
\operatorname{Var}\left(\Theta_{2, n}\right)=\frac{(n-1)^{2}}{\lambda^{2}} \sum_{i=1}^{n-1} \frac{1}{i^{2}(n-i)^{2}} .
$$

For $k=3$, since

$$
p_{3, n}(i)=\frac{i}{n-1}+\frac{i}{n-2}-\frac{i^{2}}{(n-1)(n-2)}=\frac{i(2 n-3-i)}{(n-1)(n-2)},
$$

we obtain using (7)

$$
\operatorname{Var}\left(\Theta_{3, n}\right)=\frac{(n-1)^{2}(n-2)^{2}}{\lambda^{2}} \sum_{i=1}^{n-1} \frac{1}{i^{2}(n-i)^{2}(2 n-3-i)^{2}} .
$$

The limiting variance of the rumor spreading time is given in the following result.

Theorem 2. We have

$$
\lim _{n \rightarrow \infty} \operatorname{Var}\left(\Theta_{2, n}\right)=\frac{\pi^{2}}{3 \lambda^{2}} \text { and } \lim _{n \rightarrow \infty} \operatorname{Var}\left(\Theta_{3, n}\right)=\frac{5 \pi^{2}}{24 \lambda^{2}} \text {. }
$$

Proof. For $k=2$, we have from Relation (8)

$$
\begin{aligned}
\operatorname{Var}\left(\Theta_{2, n}\right)= & \frac{(n-1)^{2}}{\lambda^{2} n^{2}}\left(\sum_{i=1}^{n-1} \frac{1}{i^{2}}+\sum_{i=1}^{n-1} \frac{1}{(n-i)^{2}}\right. \\
& \left.+2 \sum_{i=1}^{n-1} \frac{1}{i(n-i)}\right) \\
= & \frac{(n-1)^{2}}{\lambda^{2} n^{2}}\left(2 \sum_{i=1}^{n-1} \frac{1}{i^{2}}+\frac{2 H_{n-1}}{n}\right) .
\end{aligned}
$$


By taking the limit when $n$ tends to infinity, we get the first result.

For $k=3$, we have from Relation (6) and (9),

$$
\operatorname{Var}\left(\Theta_{3, n}\right)=\frac{1}{\lambda^{2}} \sum_{i=1}^{n-1}\left(\frac{A_{n}}{i}+\frac{B_{n}}{n-i}+\frac{C_{n}}{2 n-3-i}\right)^{2},
$$

where the $A_{n}, B_{n}$ and $C_{n}$ and their limits are given in the proof of Theorem 1 . This leads to

$$
\begin{aligned}
& \operatorname{Var}\left(\Theta_{3, n}\right)=\frac{1}{\lambda^{2}}\left(A_{n}^{2} \sum_{i=1}^{n-1} \frac{1}{i^{2}}+B_{n}^{2} \sum_{i=1}^{n-1} \frac{1}{i^{2}}+C_{n}^{2} \sum_{i=n-2}^{2 n-4} \frac{1}{i^{2}}\right. \\
& +2 A_{n} B_{n} \sum_{i=1}^{n-1} \frac{1}{i(n-i)}+2 A_{n} C_{n} \sum_{i=1}^{n-1} \frac{1}{i(2 n-3-i)} \\
& \left.+2 B_{n} C_{n} \sum_{i=1}^{n-1} \frac{1}{(n-i)(2 n-3-i)}\right) .
\end{aligned}
$$

Observing that

$$
\begin{aligned}
& \lim _{n \rightarrow \infty} \sum_{i=n-2}^{2 n-4} \frac{1}{i^{2}}=0, \quad \sum_{i=1}^{n-1} \frac{1}{i(n-i)}=\frac{2 H_{n-1}}{n}, \\
& \sum_{i=1}^{n-1} \frac{1}{i(2 n-3-i)} \leq \frac{H_{n-1}}{n-2}, \text { and } \\
& \sum_{i=1}^{n-1} \frac{1}{(n-i)(2 n-3-i)} \leq \frac{H_{n-1}}{n-2},
\end{aligned}
$$

we obtain $\lim _{n \rightarrow \infty} \operatorname{Var}\left(\Theta_{3, n}\right)=\frac{1}{\lambda^{2}}\left(\frac{\pi^{2}}{24}+\frac{\pi^{2}}{6}\right)=\frac{5 \pi^{2}}{24 \lambda^{2}}$, which completes the proof.

Figure 2 illustrates the standard deviations of respectively $\Theta_{2, n}$ (square root of Relation 8) and $\Theta_{3, n}$ (square root of Relation 9. It is worth observing that in both cases the standard deviation converges very quickly to its limit and that the standard deviation of $\Theta_{3, n}$ is smaller than the standard deviation of $\Theta_{2, n}$.

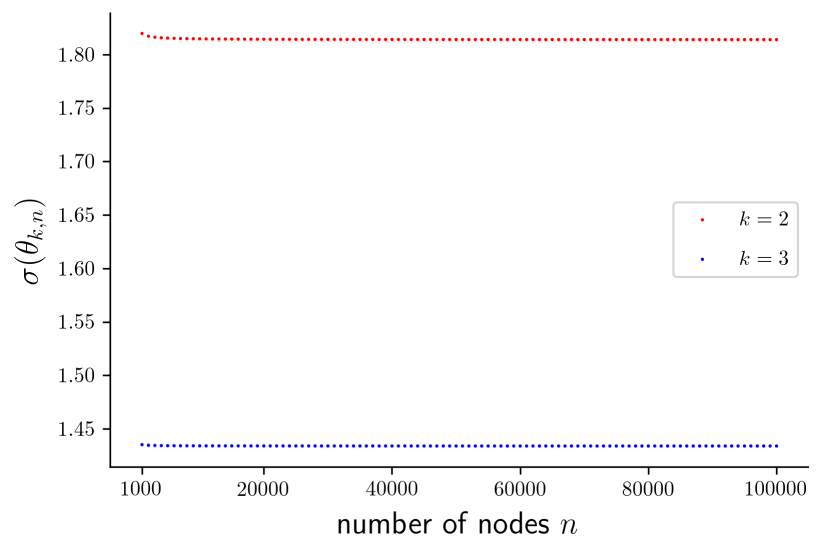

Fig. 2. Standard deviations $\sigma\left(\Theta_{2, n}\right)$ and $\sigma\left(\Theta_{3, n}\right)$ as a function of the number of nodes $n$.

\section{Distribution OF THE RUMOR SPREADING TIME}

This section provides the explicit limiting distribution of $\Theta_{k, n}-\mathbb{E}\left(\Theta_{k, n}\right)$ for $k \in\{2,3\}$. Note that the hypothesis of the principle of accompanying laws of Theorem 3.1.14 of [30], which is used in [28], is no more valid in the continuous-time case. That is why we need to use the following different proofs.

Theorem 3. If $Z_{1}$ and $Z_{2}$ are two independent random variables exponentially distributed with rate 1 then we have

$$
\Theta_{2, n}-\frac{2 \ln (n)}{\lambda} \stackrel{\mathcal{L}}{\longrightarrow}-\frac{1}{\lambda}\left(\ln \left(Z_{1}\right)+\ln \left(Z_{2}\right)\right) \text { as } n \longrightarrow \infty
$$

and

$$
\Theta_{3, n}-\frac{3 \ln (n)}{2 \lambda} \stackrel{\mathcal{L}}{\longrightarrow}-\frac{1}{\lambda}\left(\frac{\ln \left(Z_{1}\right)}{2}+\ln \left(Z_{2}\right)+\frac{\ln (2)}{2}\right)
$$

as $n \longrightarrow \infty$.

Proof. We introduce the notation $\mu_{k, n}(i)=\lambda(n-i) p_{k, n}(i)$. Since $U_{k, n}(i)$ is exponentially distributed with rate $\mu_{k, n}(i)$, it follows that for every $x \geq 0$, we have

$$
\mathbb{P}\left\{\mu_{k, n}(i) U_{k, n}(i)>x\right\}=\mathbb{P}\left\{U_{k, n}(i)>x / \mu_{k, n}(i)\right\}=e^{-x} .
$$

This means that if $Z_{i}$ is a random variable exponentially distributed with rate 1 , then we have

$$
\mu_{k, n}(i) U_{k, n}(i) \stackrel{\mathcal{L}}{=} Z_{i} .
$$

Moreover since the $\left(U_{k, n}(i)\right)_{i=1, \ldots, n-1}$ are independent, the $\left(Z_{i}\right)_{i \geq 1}$ are also independent.

We thus have for $k=2$, since $\mu_{2, n}(i)=\lambda i(n-i) /(n-1)$,

$$
\begin{aligned}
\Theta_{2, n}- & \frac{2}{\lambda} \\
= & \sum_{i=1}^{n-1} U_{2, n}(i)-\frac{2 \ln (n)}{\lambda} \\
& \underline{\underline{L}} \sum_{i=1}^{n-1} \frac{Z_{i}}{\mu_{2, n}(i)}-\frac{2 \ln (n)}{\lambda} \\
= & \frac{1}{\lambda}\left[(n-1) \sum_{i=1}^{n-1} \frac{Z_{i}}{i(n-i)}-2 \ln (n)\right] \\
= & \frac{1}{\lambda}\left[\frac{(n-1)}{n}\left(\sum_{i=1}^{n-1} \frac{Z_{i}}{i}+\sum_{i=1}^{n-1} \frac{Z_{i}}{n-i}\right)-2 \ln (n)\right] \\
= & \frac{1}{\lambda}\left[\frac{(n-1)}{n}\left(\sum_{i=1}^{n-1} \frac{Z_{i}-1}{i}+\sum_{i=1}^{n-1} \frac{Z_{n-i}-1}{i}\right)\right. \\
& \left.+2\left(\frac{(n-1) H_{n-1}}{n}-\ln (n)\right)\right]
\end{aligned}
$$

Because, as shown by L. Gordon in [19], $\sum_{i=1}^{\infty}\left(Z_{i}-1\right) / i<\infty$ P-a.s., we have

$$
\begin{aligned}
\sum_{i=1}^{n-1} \frac{Z_{i}-1}{i} & =\sum_{i=1}^{n / 2} \frac{Z_{i}-1}{i}+\sum_{i=n / 2+1}^{n-1} \frac{Z_{i}-1}{i} \\
& =\sum_{i=1}^{n / 2} \frac{Z_{i}-1}{i}+o(1) .
\end{aligned}
$$


In the same way, we have

$$
\begin{aligned}
\sum_{i=1}^{n-1} \frac{Z_{n-i}-1}{i} & =\sum_{i=1}^{n / 2-1} \frac{Z_{n-i}-1}{i}+\sum_{i=n / 2}^{n-1} \frac{Z_{n-i}-1}{i} \\
& \underline{\mathcal{L}} \sum_{i=1}^{n / 2-1} \frac{\widetilde{Z}_{i}-1}{i}+\sum_{i=n / 2}^{n-1} \frac{\widetilde{Z}_{i}-1}{i} \\
& =\sum_{i=1}^{n / 2-1} \frac{\widetilde{Z}_{i}-1}{i}+o(1),
\end{aligned}
$$

where $\widetilde{Z}_{i}=Z_{n-i}$ is exponentially distributed with rate 1 . We thus obtain

$$
\begin{aligned}
& \Theta_{2, n}-\frac{2 \ln (n)}{\lambda} \stackrel{\mathcal{L}}{=} \\
& \frac{1}{\lambda}\left[\alpha_{2, n}+2\left(\frac{(n-1) H_{n-1}}{n}-\ln (n)\right)+o(1)\right],
\end{aligned}
$$

where

$$
\alpha_{2, n}=\frac{n-1}{n}\left(\sum_{i=1}^{n / 2} \frac{Z_{i}-1}{i}+\sum_{i=1}^{n / 2-1} \frac{\widetilde{Z}_{i}-1}{i}\right)
$$

Observe that the $Z_{i}$ and the $\widetilde{Z}_{i}$ involved in $\alpha_{k, n}$ are all independent and exponentially distributed with rate 1 . Using the result of L. Gordon in [19] which states that $\sum_{i=1}^{\infty}\left(Z_{i}-\right.$ $1) / i \stackrel{\mathcal{L}}{=}-\gamma-\ln \left(Z_{1}\right)$, we get

$$
\alpha_{2, n} \stackrel{\mathcal{L}}{\longrightarrow}-\ln \left(Z_{1}\right)-\ln \left(Z_{2}\right)-2 \gamma \text { as } n \longrightarrow \infty
$$

where $\gamma$ is the Euler-Mascheroni constant. It follows from 10 that

$$
\begin{aligned}
\lim _{n \rightarrow \infty} \mathbb{P} & \left\{\Theta_{2, n}-\frac{2 \ln (n)}{\lambda}>x\right\}= \\
\mathbb{P} & \left\{-\frac{1}{\lambda}\left(\ln \left(Z_{1}\right)+\ln \left(Z_{2}\right)\right)>x\right\} .
\end{aligned}
$$

For $k=3$ we have $\mu_{3, n}(i)=\lambda i(n-i)(2 n-3-i) /((n-$ 1) $(n-2))$, which gives

$$
\begin{aligned}
& \Theta_{3, n}-\frac{3 \ln (n)}{2 \lambda}=\sum_{i=1}^{n-1} U_{3, n}(i)-\frac{3 \ln (n)}{2 \lambda} \\
& \stackrel{\mathcal{L}}{=} \sum_{i=1}^{n-1} \frac{Z_{i}}{\mu_{3, n}(i)}-\frac{3 \ln (n)}{2 \lambda} \\
& =\frac{1}{\lambda}\left[(n-1)(n-2) \sum_{i=1}^{n-1} \frac{Z_{i}}{i(n-i)(2 n-3-i)}-\frac{3 \ln (n)}{2}\right] .
\end{aligned}
$$

Using Relation (6), we obtain

$$
\begin{aligned}
& \Theta_{3, n}-\frac{3 \ln (n)}{2 \lambda} \\
& \stackrel{\mathcal{L}}{=} \frac{1}{\lambda}\left[A_{n} \sum_{i=1}^{n-1} \frac{Z_{i}}{i}+B_{n} \sum_{i=1}^{n-1} \frac{Z_{i}}{n-i}+C_{n} \sum_{i=1}^{n-1} \frac{Z_{i}}{2 n-3-i}\right. \\
& \left.-\frac{3 \ln (n)}{2}\right] \\
& =\frac{1}{\lambda}\left[A_{n} \sum_{i=1}^{n-1} \frac{Z_{i}-1}{i}+A_{n} H_{n-1}+B_{n} \sum_{i=1}^{n-1} \frac{Z_{n-i}-1}{i}\right. \\
& +B_{n} H_{n-1}+C_{n} \sum_{i=n-2}^{2 n-4} \frac{Z_{2 n-3-i}-1}{i} \\
& \left.+C_{n} \sum_{i=n-2}^{2 n-4} \frac{1}{i}-\frac{3 \ln (n)}{2}\right]
\end{aligned}
$$

where the $A_{n}, B_{n}$ and $C_{n}$ and their limits are given in the proof of Theorem 11. As we did for the case $k=2$, we can write

$$
\begin{aligned}
\Theta_{3, n}- & \frac{3 \ln (n)}{2 \lambda} \stackrel{\mathcal{L}}{=} \frac{1}{\lambda}\left[A_{n} \sum_{i=1}^{n / 2} \frac{Z_{i}-1}{i}+B_{n} \sum_{i=1}^{n / 2} \frac{\widetilde{Z}_{i}-1}{i}\right. \\
& +C_{n} \sum_{i=n-2}^{2 n-4} \frac{Z_{2 n-3-i}-1}{i}+o(1)+A_{n} H_{n-1} \\
& \left.+B_{n} H_{n-1}+C_{n}\left(H_{2 n-4}-H_{n-3}\right)-\frac{3 \ln (n)}{2}\right],
\end{aligned}
$$

where $\widetilde{Z}_{i}=Z_{n-i}$ is exponentially distributed with rate 1 . Observe that

$$
\sum_{i=n-2}^{2 n-4} \frac{Z_{2 n-3-i}-1}{i} \stackrel{\mathcal{L}}{=} \sum_{i=n-2}^{2 n-4} \frac{\hat{Z}_{i}-1}{i}
$$

where $\hat{Z}_{i}=Z_{2 n-3-i}$ is exponentially distributed with rate 1. Since $\sum_{i=1}^{\infty}\left(Z_{i}-1\right) / i<\infty$ P-a.s., we have $\sum_{i=n-2}^{2 n-4}\left(\hat{Z}_{i}-1\right) / i=o(1)$. Moreover, since the limit of $C_{n}\left(H_{2 n-4}-H_{n-3}\right)$ when $n$ tends to infinity is equal to $-\ln (2) / 2$ and since

$$
\begin{aligned}
A_{n} \sum_{i=1}^{n / 2} \frac{Z_{i}-1}{i} & +B_{n} \sum_{i=1}^{n / 2} \frac{\widetilde{Z}_{i}-1}{i} \stackrel{\mathcal{L}}{\longrightarrow} \\
& -\frac{\ln \left(Z_{1}\right)}{2}-\ln \left(Z_{2}\right)-\frac{3 \gamma}{2} \text { as } n \longrightarrow \infty,
\end{aligned}
$$

we finally get

$$
\Theta_{3, n}-\frac{3 \ln (n)}{2 \lambda} \stackrel{\mathcal{L}}{\longrightarrow}-\frac{1}{\lambda}\left(\frac{\ln \left(Z_{1}\right)}{2}+\ln \left(Z_{2}\right)+\frac{\ln (2)}{2}\right),
$$

or equivalently

$$
\begin{aligned}
\lim _{n \rightarrow \infty} \mathbb{P}\left\{\Theta_{3, n}-\frac{3 \ln (n)}{2 \lambda}>x\right\}= \\
\mathbb{P}\left\{-\frac{1}{\lambda}\left(\frac{\ln \left(Z_{1}\right)}{2}+\ln \left(Z_{2}\right)+\frac{\ln (2)}{2}\right)>x\right\},
\end{aligned}
$$


which completes the proof

The following corollary gives the explicit limiting distribution of the random variables $\Theta_{2, n}-2 \ln (n) / \lambda$ and $\Theta_{3, n}-$ $3 \ln (n) /(2 \lambda)$ when $n$ tends to infinity.

Corollary 1. For all $x \in \mathbb{R}$, we have

$$
\begin{aligned}
& \lim _{n \longrightarrow \infty} \mathbb{P}\left\{\Theta_{2, n}-\frac{2 \ln (n)}{\lambda} \leq x\right\}= \\
& \int_{0}^{\infty} \exp \left(-t-t^{-1} e^{-\lambda x}\right) d t=2 e^{-\lambda x / 2} K_{1}\left(2 e^{-\lambda x / 2}\right),
\end{aligned}
$$

where $K_{1}$ is the modified Bessel function of the second kind of order 1 given, for $z>0$, by

$$
K_{1}(z)=\frac{z}{4} \int_{0}^{+\infty} t^{-2} e^{-t-z^{2} / 4 t} d t
$$

and

$$
\begin{aligned}
& \lim _{n \rightarrow \infty} \mathbb{P}\left\{\Theta_{3, n}-\frac{3 \ln (n)}{2 \lambda} \leq x\right\}= \\
& \int_{0}^{\infty} \exp \left(-t-t^{-2} e^{-2 \lambda x} / 2\right) d t .
\end{aligned}
$$

Proof. For $k=2$, using Theorem 3 , we have, for all $x \in \mathbb{R}$,

$$
\begin{aligned}
\lim _{n \longrightarrow \infty} \mathbb{P}\left\{\Theta_{2, n}-\frac{2 \ln (n)}{\lambda}\right. & \leq x\}= \\
& \mathbb{P}\left\{-\frac{1}{\lambda}\left(\ln \left(Z_{1}\right)+\ln \left(Z_{2}\right)\right) \leq x\right\} .
\end{aligned}
$$

The variables $Z_{1}$ and $Z_{2}$ being independent and exponentially distributed with rate 1 , we obtain

$$
\begin{aligned}
\mathbb{P}\left\{-\frac{1}{\lambda}\left(\ln \left(Z_{1}\right)+\ln \left(Z_{2}\right)\right)\right. & \leq x\}=\mathbb{P}\left\{Z_{1} Z_{2} \geq e^{-\lambda x}\right\} \\
& =\int_{0}^{\infty} \mathbb{P}\left\{Z_{1} \geq t^{-1} e^{-\lambda x}\right\} e^{-t} d t \\
& =\int_{0}^{\infty} \exp \left(-t-t^{-1} e^{-\lambda x}\right) d t
\end{aligned}
$$

which can be written as $2 e^{-\lambda x / 2} K_{1}\left(2 e^{-\lambda x / 2}\right)$. In the same way, using Theorem 3 , we have, for all $x \in \mathbb{R}$,

$$
\begin{aligned}
\lim _{n \rightarrow \infty} \mathbb{P}\left\{\Theta_{3, n}-\frac{3 \ln (n)}{2 \lambda} \leq x\right\}= \\
\quad \mathbb{P}\left\{-\frac{1}{\lambda}\left(\frac{\ln \left(Z_{1}\right)}{2}+\ln \left(Z_{2}\right)+\frac{\ln (2)}{2}\right) \leq x\right\} .
\end{aligned}
$$

The variables $Z_{1}$ and $Z_{2}$ being independent and exponentially distributed with rate 1 , we obtain

$$
\begin{aligned}
\mathbb{P}\left\{-\frac{1}{\lambda}\left(\frac{\ln \left(Z_{1}\right)}{2}+\right.\right. & \left.\left.\ln \left(Z_{2}\right)+\frac{\ln (2)}{2}\right) \leq x\right\} \\
& =\mathbb{P}\left\{2^{1 / 2} Z_{1}^{1 / 2} Z_{2} \geq e^{-\lambda x}\right\} \\
& =\int_{0}^{\infty} \mathbb{P}\left\{Z_{1} \geq t^{-2} e^{-2 \lambda x} / 2\right\} e^{-t} d t \\
& =\int_{0}^{\infty} \exp \left(-t-t^{-2} e^{-2 \lambda x} / 2\right) d t
\end{aligned}
$$

which completes the proof.

Introducing the functions $F_{2}$ and $F_{3}$ defined, for $x \geq 0$, by

$$
F_{2}(x)=\lim _{n \longrightarrow \infty} \mathbb{P}\left\{\left|\Theta_{2, n}-\frac{2 \ln (n)}{\lambda}\right| \leq x\right\}
$$

and

$$
F_{3}(x)=\lim _{n \longrightarrow \infty} \mathbb{P}\left\{\left|\Theta_{3, n}-\frac{3 \ln (n)}{2 \lambda}\right| \leq x\right\} .
$$

While the Bienaym-Tchebychev (BT) inequality only gives a bound of these functions, our result gives their exact values. Indeed, this inequality gives, for $x>0$ and large values of $n, F_{2}(x) \geq 1-\pi^{2} /\left(3 \lambda x^{2}\right)$. For instance, for $\lambda=1$ and $x=\pi^{2} / 3$, the BT inequality only gives $F_{2}(x) \geq 0$ while our result gives $F_{2}(x)=0.8798042582$, that is

$$
\lim _{n \longrightarrow \infty} \mathbb{P}\left\{2 \ln (n)-\frac{\pi^{2}}{3} \leq \Theta_{2, n} \leq 2 \ln (n)+\frac{\pi^{2}}{3}\right\}=0.8798 .
$$

The same observation is clearly also valid for function $F_{3}$ and for the functions $G_{2}$ and $G_{3}$ defined, for $x \geq 0$, by

$$
G_{2}(x)=\lim _{n \longrightarrow \infty} \mathbb{P}\left\{\mid \Theta_{2, n}-\mathbb{E}\left(\Theta_{2, n} \mid \leq x\right\}\right.
$$

and

$$
G_{3}(x)=\lim _{n \longrightarrow \infty} \mathbb{P}\left\{\left|\Theta_{3, n}-\mathbb{E}\left(\Theta_{3, n}\right)\right| \leq x\right\} .
$$

The values of these four functions are illustrated in Figure 3

The following corollary gives the explicit limiting distribution of the random variables $\Theta_{2, n}-\mathbb{E}\left(\Theta_{2, n}\right)$ and $\Theta_{3, n}-$ $\mathbb{E}\left(\Theta_{3, n}\right)$ when $n$ tends to infinity.

Corollary 2. For all $x \in \mathbb{R}$, we have

$$
\lim _{n \longrightarrow \infty} \mathbb{P}\left\{\Theta_{2, n}-\mathbb{E}\left(\Theta_{2, n}\right) \leq x\right\}=2 e^{-\lambda x / 2-\gamma} K_{1}\left(2 e^{-\lambda x / 2-\gamma}\right)
$$

and

$$
\lim _{n \longrightarrow \infty} \mathbb{P}\left\{\Theta_{3, n}-\mathbb{E}\left(\Theta_{3, n}\right) \leq x\right\}=
$$

$$
\int_{0}^{\infty} \exp \left(-t-t^{-2} e^{-2 \lambda x-3 \gamma}\right) d t
$$

Proof. For $k=2$, we have

$$
\begin{aligned}
\mathbb{P}\left\{\Theta_{2, n}-\right. & \left.\mathbb{E}\left(\Theta_{2, n}\right) \leq x\right\}= \\
& \mathbb{P}\left\{\Theta_{2, n}-\frac{2 \ln (n)}{\lambda} \leq x+\mathbb{E}\left(\Theta_{2, n}\right)-\frac{2 \ln (n)}{\lambda}\right\} .
\end{aligned}
$$

From Theorem 1 and Corollary 1, by taking the limit when $n$ tends to infinity, we obtain

$\lim _{n \rightarrow \infty} \mathbb{P}\left\{\Theta_{2, n}-\mathbb{E}\left(\Theta_{2, n}\right) \leq x\right\}=2 e^{-\lambda x / 2-\gamma} K_{1}\left(2 e^{-\lambda x / 2-\gamma}\right)$.

In the same way, for $k=3$, we have

$$
\begin{aligned}
\mathbb{P}\left\{\Theta_{3, n}-\right. & \left.\mathbb{E}\left(\Theta_{3, n}\right) \leq x\right\}= \\
& \mathbb{P}\left\{\Theta_{3, n}-\frac{3 \ln (n)}{2 \lambda} \leq x+\mathbb{E}\left(\Theta_{3, n}\right)-\frac{3 \ln (n)}{2 \lambda}\right\} .
\end{aligned}
$$


From Theorem 1 and Corollary 1 , by taking the limit when $n$ tends to infinity, we obtain

$$
\begin{aligned}
\lim _{n \rightarrow \infty} \mathbb{P}\left\{\Theta_{3, n}-\mathbb{E}\left(\Theta_{3, n}\right) \leq x\right\} & = \\
& \int_{0}^{\infty} \exp \left(-t-t^{-2} e^{-2 \lambda x-3 \gamma}\right) d t,
\end{aligned}
$$

which completes the proof.

Fig. 3. Limiting distribution of $\left|\Theta_{2, n}-\mathbb{E}\left(\Theta_{2, n}\right)\right|,\left|\Theta_{2, n}-2 \ln (n) / \lambda\right|$, $\left|\Theta_{3, n}-\mathbb{E}\left(\Theta_{3, n}\right)\right|$ and $\left|\Theta_{3, n}-3 \ln (n) / 2 \lambda\right|$ when $n$ tends to infinity, with $\lambda=1$.

\section{CONCLUSION}

In this paper, we have analysed a new rumor spreading protocol that allows each node to asynchronously interact with 2 other random nodes during each pull operation. We have shown that this protocol generalizes the standard pull protocol, in which 2 nodes interact, and improves it. Further research consists in considering the more general case where each node interacts with $k-1$ other nodes during each operation, with $k>3$.

\section{REFERENCES}

[1] H. Acan, A. Collevecchio, A. Mehrabian, and N. Wormald. On the push \& pull protocol for rumour spreading. Trends in Mathematics, 6:3-10, 2017.

[2] D. Anderson and T. Kurtz. Stochastic analysis of biochemical systems. Springer,, 2015.

[3] F. Chierichetti, S. Lattanzi, and A. Panconesi. Rumor spreading in social networks. Theoretical Computer Science, 412(24):2602-2610, 2011.

[4] A. Clementi, P. Crescenzi, C. Doerr, P. Fraigniaud, F. Pasquale, and R. Silvestri. Rumor spreading in random evolving graphs. Random structures and Algorithms, 48(2):290-312, 2015.

[5] J. Correa, M. Kiwi, N. Olver, and A. Vera. Adaptive rumor spreading. WINE 2015, page 272285, 2016.

[6] D. J. Daley and J. Gani. Epidemic Modelling: An Introduction. Cambridge Studies in Mathematical Biology. Cambridge University Press, 1999.

[7] S. Daum, F. Kuhn, and Y. Maus. Rumor spreading with bounded indegree. In Proceedings of the International Colloquium on Structural Information and Communication Complexity (SIROCCO), 2016.

[8] A. J. Demers, D. H. Greene, C. H. Hauser, W. Irish, J. Larson, S. J. Shenker, H. E. Sturgis, D. C. Swinehart, and D. B. Terry. Epidemic algorithms for replicated database maintenance. PODC 87 , pages $1-12$, 1987.
[9] B. Doerr, M. Fouz, and T. Friedrich. Asynchronous rumor spreading in preferential attachment graphs. SWAT 2012, pages 307-315, 2012.

[10] B. Doerr, M. Fouz, and T. Friedrich. Experimental analysis of rumor spreading in social networks. MedAlg 2012, pages 159-173, 2012.

[11] B. Doerr and A. Kostrygin. Randomized Rumor Spreading Revisited. In Ioannis Chatzigiannakis, Piotr Indyk, Fabian Kuhn, and Anca Muscholl, editors, ICALP 2017, volume 80, pages 138:1-138:14. Schloss Dagstuhl-Leibniz-Zentrum fuer Informatik, 2017.

[12] Z. Duan, K. Gopalan, and Y. Dong. Probabilistic reliable dissemination in large-scale systems. SRUTI, 2005.

[13] F. Feige, D. Peleg, P. Raghavan, and E. Upfal. Randomized broadcast in networks. Random Structures and Algorithms, 1(4):447-460, 1990.

[14] N. Fountoulakis and K. Panagiotou. Rumor spreading on random regular graphs and expanders. Random Structures and Algorithms, 43(2):201$220,2013$.

[15] A. Frieze and G. Grimmet. The shortest-path problem for graphs with random arc-lengths. Discrete Applied Mathematics, 10(1):57-77, 1985.

[16] G. Giakkoupis. Tight bounds for rumor spreading in graphs of a given conductance. In Proceedings of the International Symposium on Theoretical Aspects of Computer Science (STACS), 2011.

[17] G. Giakkoupis. Tight bounds for rumor spreading with vertex expansion. In Proceedings of the Annual ACM-SIAM Symposium on Discrete Algorithms (SODA), 2014.

[18] G. Giakkoupis, Y. Nazari, and P. Woelfel. How asynchrony affects rumor spreading time. PODC '16, pages 185-194, 2016.

[19] L. Gordon. Bounds for the distribution of the generalized variance. The Annals of Statistics, 17(4):1684-1692, 1989.

[20] R. Karp, C. Schindelhauer, S. Shenker, and B. Vocking. Randomized rumor spreading. In Proceedings of the Annual Symposium on Foundations of Computer Science (FOCS), 2000.

[21] T. M. Liggett. Interacting Particle Systems. Springer, New York, 1985.

[22] Y. Mocquard, S. Robert, B. Sericola, and E. Anceaume. Analysis of the propagation time of a rumour in large-scale distributed systems. NCA 2016, 2016.

[23] Y. Moreno, M. Nekovee, and A. F. Pacheco. Dynamics of rumor spreading in complex networks. Phys. Rev., 69:248-258, 2004.

[24] K. Panagiotou, X. Perez-Gimenez, T. Sauerwald, and H. Sun. Randomized rumor spreading: the effect of the network topology. Combinatorics, Probability and Computing, 24(2):457-479, 2015.

[25] K. Panagiotou, A. Pourmiri, and T. Sauerwald. Faster rumor spreading with multiple calls. The electronic journal of combinatorics, 22, 2015.

[26] B. Pittel. On spreading a rumor. SIAM Journal on Applied Mathematics, 47(1):213-223, 1987.

[27] A. Pourmiri and F. Ramezani. Brief announcement: Ultra-fast asynchronous randomized rumor spreading. SPAA 2019, 2019.

[28] F. Robin, B. Sericola, E. Anceaume, and Y. Mocquard. Stochastic analysis of rumor spreading with $k$-pull operations. Research report, 2021.

[29] S. Sanghavi, B. Hajek, and L. Massouli. Gossiping with multiple messages. IEEE Transactions on Information Theory, 53(123), 2007.

[30] D. W. Stroock. Probability Theory: An Analytic View. Cambridge University Press, second edition, 2010.

[31] G. Yao, J. Bi, S. Wang, Y. Zhang, and Y. Li. A pull model IPv6 duplicate address detection. LCN 2010, 2010. 